\title{
Expression of microRNA-10a, microRNA-342-3p and their predicted target gene TIAM1 in extranodal NK/T-cell lymphoma, nasal type
}

\author{
HAOBO HUANG ${ }^{1}$, LIPING FAN ${ }^{1}$, RONG ZHAN ${ }^{2}$, SHUNQUAN WU ${ }^{2}$ and WENYAN NIU ${ }^{2}$ \\ ${ }^{1}$ Department of Blood Transfusion, Fujian Medical University Union Hospital; ${ }^{2}$ Department of Hematology, \\ Fujian Medical University Union Hospital and Fujian Institute of Hematology, Fuzhou, Fujian 350001, P.R. China
}

Received November 23, 2014; Accepted September 14, 2015

DOI: 10.3892/ol.2015.3831

\begin{abstract}
MicroRNAs (miRNAs) may act as oncogenes or tumor suppressor genes in different types of human cancer. T-lymphoma invasion and metastasis inducing factor 1 (TIAM1) participates in the development of several types of human cancer. However, the expression of miRNAs and TIAM1 in extranodal natural killer (NK)/T-cell lymphoma, nasal type (ENKTCL) remains poorly understood. In the present study, the association between the expression levels of miR-10a and miR-342-3p and the protein expression levels of TIAM1 was examined in ENKTCL tissues. The expression levels of miR-10a, miR-22, miR-340, miR-342-3p and miR-590-5p in 15 primary ENKTCL tissues were analyzed using quantitative polymerase chain reaction, and the protein expression levels of TIAM1 in 21 primary ENKTCL tissues were analyzed using immunohistochemistry. The expression levels of miR-10a and miR-342-3p were lower in ENKTCL tissues than in normal NK cells, but no significant differences were observed in the expression levels of miR-22, miR-340 and miR-590-5p in ENKTCL tissues, compared with normal NK cells. The low expression levels of miR-10a detected in the tissues of patients with ENKTCL were inversely correlated with the age of the patients, whereas the low expression levels of miR-342-3p measured in these samples were not correlated with any demographic or clinical features of the patients. The protein expression levels of TIAM1 were higher in ENKTCL tissues than in normal and reactive lymph node hyperplasia tissues, and positively correlated with the Ann Arbor stage and international prognostic index score of the tumors. Furthermore, the expression levels of miRNA-10a and miRNA-342-3p
\end{abstract}

Correspondence to: Professor Rong Zhan, Department of Hematology, Fujian Medical University Union Hospital and Fujian Institute of Hematology, 29 Xinquan Road, Gulou, Fuzhou, Fujian 350001, P.R. China

E-mail: huanghaobo1981@163.com

Key words: extranodal NK/T-cell lymphoma, nasal type, miR-10a, miR-342-3p, TIAM1 were inversely correlated with the protein expression levels of TIAM1 in ENKTCL tissues. These data suggest that TIAM1 may contribute to the pathogenesis of ENKTCL, and miRNA-10a and miRNA-342-3p may be involved in the development of ENKTCL via the TIAM1 pathway.

\section{Introduction}

Extranodal natural killer (NK)/T-cell lymphoma, nasal type (ENKTCL) is an aggressive type of lymphoma that occurs frequently in the Asian population $(1,2)$. The pathogenesis of this tumor is poorly understood, and conventional chemotherapy regimens, currently employed for the treatment of other aggressive types of lymphoma, usually provide poor outcomes in patients with ENKTCL $(1,2)$. Therefore, studies on the pathogenetic abnormalities that occur during the development of ENKTCL may contribute to improving the clinical outcomes of these patients.

MicroRNAs (miRNAs) are small non-coding RNA molecules that inhibit the transcription or translation of mRNA. Previous studies have demonstrated that dysregulation of miRNA occurs in numerous types of human cancer, indicating that miRNAs may act as oncogenes or tumor suppressor genes (3). Previous miRNA expression profiling studies conducted on a series of ENKTCL formalin-fixed paraffin-embedded (FFPE) tissues revealed that several miRNAs, including miR-10a, miR-22, miR-340, miR-342-3p and miR-590-5p, are dysregulated in ENKTCL tissues, compared with normal NK cells (1). Bioinformatic analysis of these miRNAs indicated that all of them target the T-lymphoma invasion and metastasis-inducing factor 1 (TIAM1) gene (1).

Tiam1 is a specific guanine nucleotide exchange factor for Rho-like guanosine triphosphate (GTP)ases, which exhibits its pathophysiological role via the activation of the rat sarcoma-related $\mathrm{C} 3$ botulinum toxin substrate signaling pathway (4). Overexpression of TIAM1 has been reported in various solid tumors (5-12). In addition, previous studies have demonstrated that TIAM1 modulates a number of cellular processes considered to be associated with tumor progression, including cell apoptosis, invasion and migration (13-18). These findings suggest that TIAM1 may be a target for cancer 
Table I. Primer sequences of miRNAs for quantitative polymerase chain reaction analysis.

Primer sequence (5'-3')

\begin{tabular}{lll}
\cline { 2 - 3 } Gene & \multicolumn{1}{c}{ Forward } & \multicolumn{1}{c}{ Reverse } \\
\hline miRNA-10a & TACCCTGTAGATCCGAATTTGTG & ATTCCCCTAGATACGAATTTGTGA \\
miRNA-22 & AGCAACATGCCCTGCTC & TCTGTCACCTTCCAGATGATG \\
miRNA-340 & ATAAAGCAATGAGACTGATTGTC & GGCTATAAAGTAACTGAGACGGA \\
miRNA-342-3p & GTGCTATCTGTGATTGAGGGA & CGGGTGCGATTTCTGTG \\
miRNA-590-5p & TTAGAGCCAACCAGCAGC & GCATTGACAGCACATCCC \\
U6 & GTTTTGTAGTTTTTGGAGTTAGTGTTGTGT & CTCAACCTACAATCAAAAACAACACAAACA
\end{tabular}

miRNA, microRNA.

therapy. However, there is limited evidence regarding the role of the TIAM1 gene in the pathogenesis of ENKTCL.

To gain insight into the potential role of miR-10a, miR-22, miR-340, miR-342-3p, miR-590-5p and TIAM1 in the pathogenesis of ENKTCL, the present study examined the expression levels of these miRNAs and their target gene TIAM1 in ENKTCL tissues, in order to assess whether the expression levels of these molecules correlated with the clinical features of patients with ENKTCL.

\section{Materials and methods}

Patients and controls. Patients who were diagnosed with ENKTCL from 2007 to 2011 were selected from the archives of the Department of Pathology of the Fujian Medical University Union Hospital (Fuzhou, China), and classified according to the 2008 World Health Organization classification of lymphomas (19). A total of 21 patients were selected for the study. The study was approved by the ethics committee of Fujian Medical University Union Hospital. The samples were collected with the patients' consent. Patients with no additional tissue available for immunohistochemical testing were excluded from the study. The FFPE tissues of 15 patients were subjected to quantitative polymerase chain reaction (qPCR) analysis. In addition, ten samples of normal and reactive lymph node hyperplasia FFPE tissue were included as controls.

Isolation of normal NK cells from peripheral blood. Human normal NK cells were isolated from whole blood samples obtained from healthy donors, which were collected with EDTA using NK Cell Isolation Kit (TBD Science, Tianjin, China).

RNA extraction. Prior to RNA extraction, five pieces of the FFPE tissue sections were treated with $1 \mathrm{ml}$ xylene (Kemiou Chemical Reagent Co., Tianjin, China) for $10 \mathrm{sec}$ at room temperature,then incubated at 56 degree for $3 \mathrm{~min}$ to remove the paraffin, and subsequently digested with $10 \mu \mathrm{l}$ proteinase $\mathrm{K}$ (Qiagen, Inc., Valencia, CA, USA), followed by incubation at $56^{\circ} \mathrm{C}$ for $15 \mathrm{~min}$, then at $80^{\circ} \mathrm{C}$ for $15 \mathrm{~min}$. Total RNA was then isolated using miRNeasy FFPE Kit (Qiagen, Inc.), according to the manufacturer's protocol. Total RNA from NK cells was extracted with TRIzol (Invitrogen, Thermo Fisher Scientific, Inc., Waltham, MA, USA).

Reverse transcription (RT) and qPCR of miRNAs. Complementary DNA was synthesized from total RNA using PrimeScript ${ }^{\mathrm{TM}}$ RT reagent Kit (Perfect Real Time) (Takara Bio, Inc., Otsu, Japan), following the manufacturer's protocol. The RT reactions contained $500 \mathrm{ng}$ total RNA extracted from the samples, $2 \mu 1$ XX PrimeScript ${ }^{\mathrm{TM}}$ Buffer (Takara Bio, Inc.), $0.5 \mu \mathrm{l} 1 \mathrm{X}$ PrimeScript ${ }^{\mathrm{TM}}$ RT Enzyme Mix I (Takara Bio, Inc.) and $0.5 \mu \mathrm{l}$ oligo(dT) primer (Takara Bio, Inc.). The 10- $\mu \mathrm{l}$ reactions were incubated for $42 \mathrm{~min}$ at $37^{\circ} \mathrm{C}$, followed by 30 -sec incubation at $85^{\circ} \mathrm{C}$, and then exposed to $4{ }^{\circ} \mathrm{C}$.

To quantify the expression levels of the aforementioned miRNAs, qPCR was conducted using SYBR ${ }^{\circledR}$ Premix Ex Taq $^{\mathrm{TM}}$ II (Tli RNase H Plus) Kit (Takara, Bio, Inc.) with an Applied Biosystems 7300 Real Time PCR System (Thermo Fisher Scientific, Inc.), according to the manufacturer's protocol. The $20-\mu \mathrm{l}$ PCR reactions included $0.19 \mu \mathrm{l}$ RT product, $10.0 \mu 12 \mathrm{X} \mathrm{SYBR}^{\circledR}$ Premix Ex Taq ${ }^{\mathrm{TM}}$ II (Takara Bio, Inc.), $2.0 \mu 1$ primers mix (Biosune, Inc., Shanghai, China), $0.4 \mu 1$ 50X ROX Reference Dye II (Takara Bio, Inc.) and $7.41 \mu 1$ RNase-free $\mathrm{dH}_{2} \mathrm{O}$ (Takara Bio, Inc.). The reaction mixtures were incubated in a 96 -well plate at $95^{\circ} \mathrm{C}$ for $1 \mathrm{~min}$, followed by 40 cycles of amplification at $95^{\circ} \mathrm{C}$ for $5 \mathrm{sec}$ and $60^{\circ} \mathrm{C}$ for $30 \mathrm{sec}$. The sequences of the primers used are listed in Table I. The quantification cycle $\left(\mathrm{C}_{\mathrm{q}}\right)$ was determined using default threshold settings. All the experiments were performed in triplicate. U6 small nuclear RNA was used as control to normalize the miRNA input in the qPCR assay. The qPCR data were analyzed using the $2^{-\triangle \mathrm{Cq}}$ method.

Immunohistochemistry. Tissue sections were subjected to antigen retrieval by incubation in $10 \mathrm{mmol} / 1$ sodium citrate buffer $(\mathrm{pH} \mathrm{6.0)}$ ) for $10 \mathrm{~min}$ in a microwave oven (WD900SL23-2, Galanz Enterprises Co., Ltd., Foshan, China) at the maximum power setting. Any potential endogenous peroxidase activity present in the tissues was blocked by incubation with $3 \% \mathrm{H}_{2} \mathrm{O}_{2}$ for $15 \mathrm{~min}$. Subsequently, the tissue sections were incubated at room temperature for 60 min with a rabbit polyclonal antibody specific for human TIAM1 (dilution, 7 1:100; cat no. sc-872, Santa Cruz Biotechnology, Inc., Dallas, TX, USA) diluted in phosphate-buffered 
Table II. Clinical features of 21 patients with ENKTCL.

\begin{tabular}{|c|c|c|c|c|c|c|c|c|}
\hline $\begin{array}{l}\text { Patient } \\
\text { no. }\end{array}$ & $\begin{array}{c}\text { Age } \\
\text { (years) }\end{array}$ & Gender & $\begin{array}{c}\text { Primary } \\
\text { cancer site }\end{array}$ & Stage $^{a}$ & $\begin{array}{l}\mathrm{LDH} \\
\text { levels }\end{array}$ & B symptoms & IPI & $\begin{array}{c}\text { TIAM1 in } \\
\text { ENKTCL }(\text { IHS })^{\mathrm{b}}\end{array}$ \\
\hline 1 & 27 & Male & Nasal cavity & I & Normal & A & 0 & 0 \\
\hline 2 & 52 & Female & Nasal cavity & II & Normal & B & 0 & 0 \\
\hline 3 & 71 & Male & Nasal cavity & I & Normal & A & 1 & 0 \\
\hline 4 & 68 & Male & Gingiva & IV & Normal & A & 4 & 0 \\
\hline 5 & 24 & Male & Adrenal gland & III & Normal & A & 2 & 2 \\
\hline 6 & 54 & Male & Nasal cavity & IV & High & B & 4 & 0 \\
\hline 7 & 55 & Male & Nasal cavity & I & Normal & A & 0 & 2 \\
\hline 8 & 45 & Female & Bone marrow & IV & Normal & B & 3 & 2 \\
\hline 9 & 39 & Male & Nasal cavity & IV & High & B & 3 & 1 \\
\hline 10 & 41 & Male & Nasal cavity & I & High & A & 1 & 2 \\
\hline 11 & 26 & Male & Gastric & IV & High & A & 4 & 0 \\
\hline 12 & 64 & Male & Nasal cavity & I & High & A & 3 & 1 \\
\hline 13 & 13 & Female & Bone marrow & IV & High & B & 4 & 2 \\
\hline 14 & 55 & Male & Bone marrow & IV & High & B & 4 & 1 \\
\hline 15 & 43 & Male & Bone marrow & IV & High & B & 4 & 0 \\
\hline 16 & 43 & Female & Nasal cavity & II & High & B & 2 & 0 \\
\hline 17 & 71 & Male & Nasal cavity & I & Normal & A & 2 & 0 \\
\hline 18 & 40 & Male & Nasal cavity & IV & Normal & B & 3 & 1 \\
\hline 19 & 39 & Male & Nasal cavity & III & Normal & B & 3 & 2 \\
\hline 20 & 24 & Male & Nasal cavity & IV & High & B & 3 & 2 \\
\hline 21 & 32 & Male & Nasal cavity & II & Normal & B & 1 & 0 \\
\hline
\end{tabular}

${ }^{a}$ Ann Arbor staging; ${ }^{b}$ protein expression levels of TIAM1 in ENKTCL tissues. ENKTCL, extranodal natural killer/T-cell lymphoma, nasal type; LDH, lactate dehydrogenase; IPI, international prognostic index; TIAM1, T-lymphoma invasion and metastasis inducing factor 1; IHS, immunohistochemistry score.

saline (PBS). Immunoreactive proteins were visualized with MaxVision ${ }^{\mathrm{TM}}$ HRP-Polymer anti-Rabbit IHC Kit (Fuzhou Maixin Biotech Co., Ltd., Fuzhou, China), following the manufacturer's protocol, and counterstained with hematoxylin (Sigma-Aldrich Shanghai Trading Co., Ltd., Shanghai, China). Tissue sections corresponding to the negative control were treated with PBS under the same experimental conditions than the samples.

Quantitative evaluation of the protein expression levels of TIAM1 was performed by counting the percentage of immunoreactive cells positive for TIAM1 that were present in a number of high-power microscopic fields (magnification, $\mathrm{x} 200$; BX41 microscope, Olympus Corporation, Tokyo, Japan). The ENKTCL tissues were scored based on the percentage of positive tumor cells expressing cytoplasmic TIAM1, as follows: i) $<15 \%$, score 0 ; ii) $15-24 \%$, score 1 ; iii) $25-49 \%$, score 2 ; iv) $50-74 \%$, score 3 ; and v) $\geq 75 \%$, score 4 .

Statistical analysis. Since the data corresponding to the expression levels of miR-10a, miR-22, miR-340, miR-342-3p and miR-590-5p followed a normal distribution, the expression levels of these miRNAs in ENKTCL tissues and normal NK cells were compared using Student's two-tailed t test. The association between the clinical features of patients with ENKTCL and the expression levels of miR-10a, miR-342-3p and TIAM1 protein detected in these patients was analyzed via Student's two-tailed t test and $\chi^{2}$ test, respectively. Spearman's rank correlation was used to evaluate the association between the expression levels of miR-10a and miR-342-3p and the protein expression levels of TIAM1. P $<0.05$ was considered to indicate a statistical significant difference. SPSS software version 17.0 (SPSS, Inc., Chicago, IL, USA) was used for statistical analysis.

\section{Results}

Clinical features of patients with ENKTCL. The main demographic and clinical features of patients with ENKTCL are listed in Table II. The median age at the time of diagnosis was 44.1 years (range, 13-71 years), and the most frequent cancer site was the nasal cavity. According to the Ann Arbor staging of lymphoma, 6 patients were in stage I, 3 in stage II, 2 in stage III and 10 in stage IV (20). High expression levels of lactate dehydrogenase (LDH) were observed in 10 patients, and 12 patients exhibited B symptoms.

qPCR results of $m i R-10 a, m i R-22, m i R-340, m i R-342-3 p$ and $m i R-590-5 p$. The expression levels of miR-10a were markedly lower in ENKTCL tissues than in normal NK cells (Fig. 1A). In addition, the expression levels of miR-342-3p in ENKTCL tissues were significantly lower than in normal NK cells (Fig. 1B). In contrast, the expression 
A

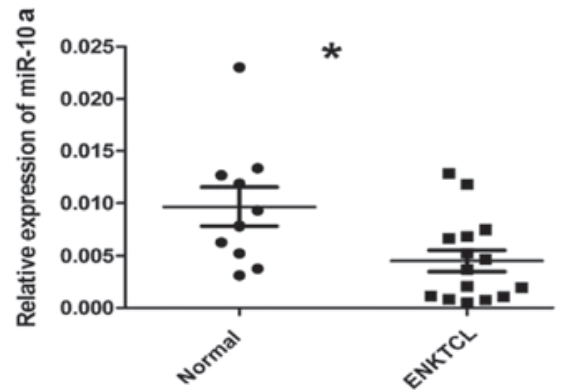

B

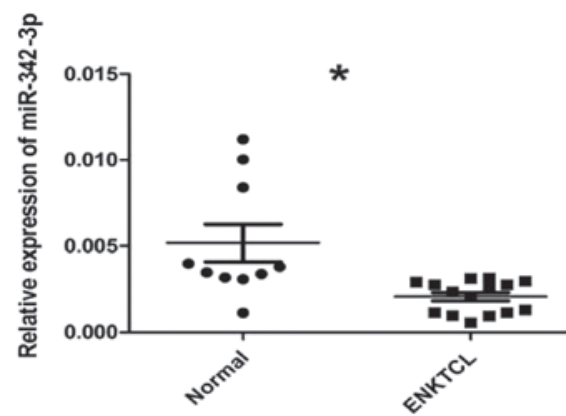

C

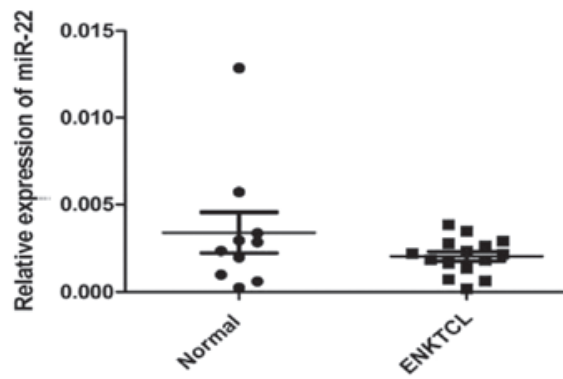

D

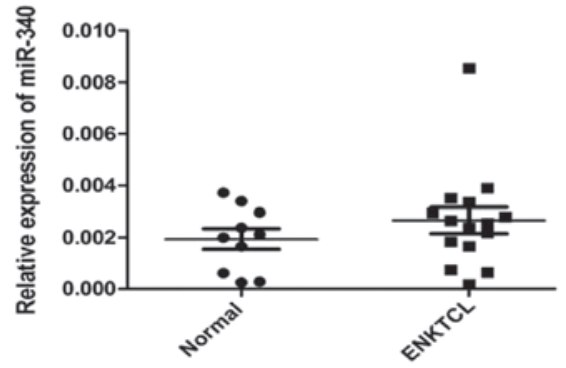

$\mathbf{E}$

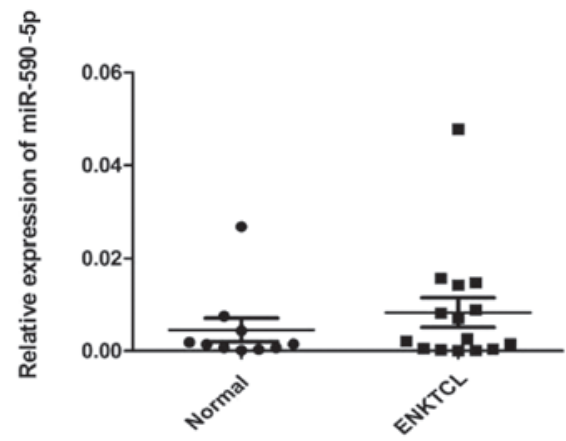

Figure 1. Expression levels of miRNAs in ENKTCL FFPE tissues and normal NK cells. The expression levels (measured relative to U6) of (A) miR-10a and (B) miR-342-3p were lower in ENKTCL tissues than in normal NK cells $\left(4.51 \pm 1.03 \times 10^{-3}\right.$ vs. $9.63 \pm 1.88 \times 10^{-3}$ and $2.08 \pm 0.24 \times 10^{-3}$ vs. $5.18 \pm 1.08 \times 10^{-3}$, respectively; ${ }^{*} \mathrm{P}<0.05$ vs. normal NK cells). The expression levels of (C) miR-22, (D) miR-340 and (E) miR-590-5p did not differ significantly between ENKTCL tissues and normal NK cells. miRNA, microRNA; ENKTCL, extranodal NK/T-cell lymphoma, nasal type; FFPE, formalin-fixed paraffin-embedded; NK, natural killer.
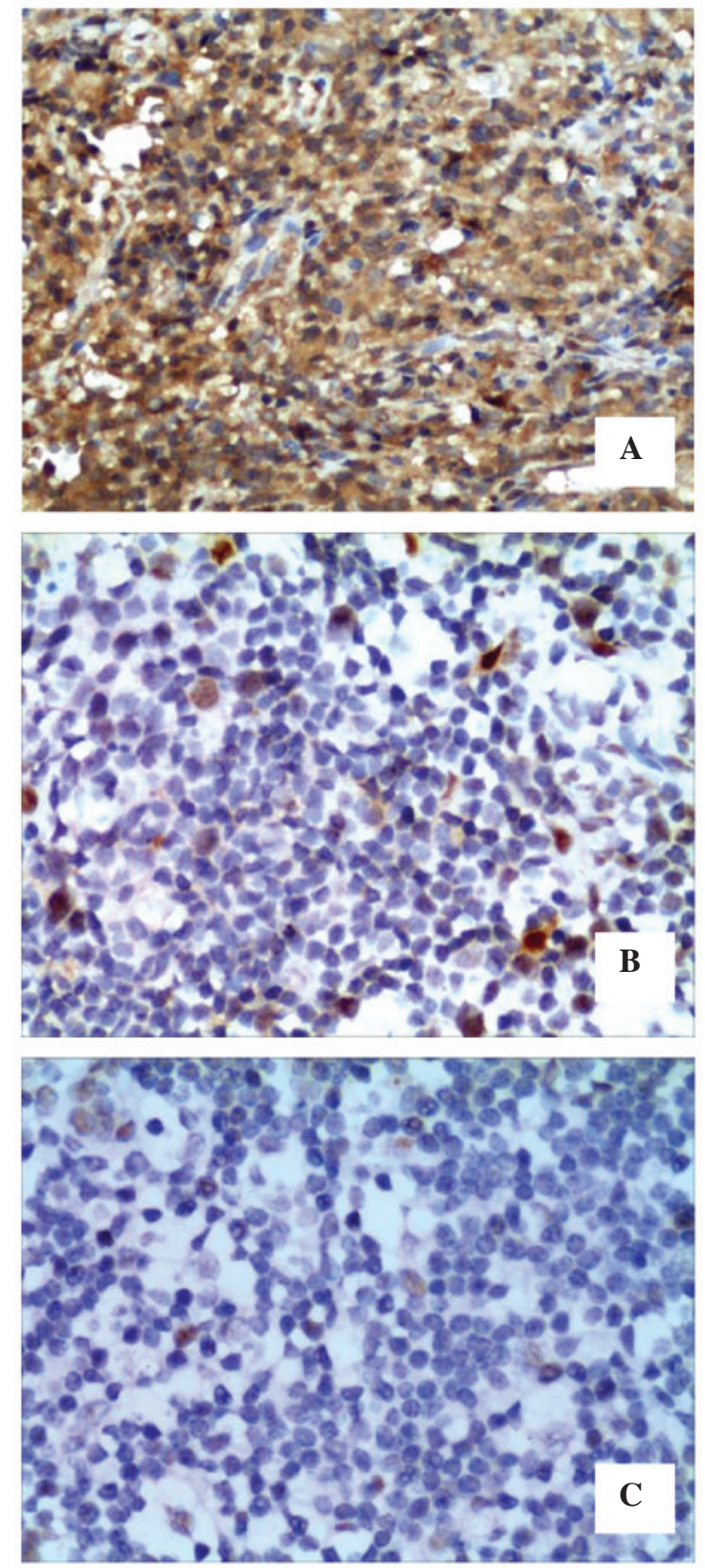

Figure 2. Immunohistochemical staining of TIAM1 in clinical FFPE tissues (magnification, $\mathrm{x} 200$ ). The rate of positive cells and the intensity of the staining for TIAM1 protein was higher in (A) extranodal natural killer/T-cell lymphoma, nasal type FFPE tissues, compared with (B) reactive lymph node hyperplasia and (C) normal lymph node tissues. TIAM1, T-lymphoma invasion and metastasis inducing factor 1; FFPE, formalin-fixed paraffin-embedded.

levels of miR-22, miR-340 and miR-590-5p did not differ significantly between ENKTCL tissues and normal NK cells (Fig. 1C-E). These results suggest that miR-10a and miR-342-3p may be involved in the pathogenesis of ENKTCL.

Correlations between the expression levels of miR-10a and $m i R-342-3 p$ and the demographic and clinical characteristics of patients with ENKTCL. The expression levels of miR-10a in ENKTCL FFPE tissues were inversely correlated with the patients' age $(\mathrm{P}=0.02)$, but not with other demographic or clinical features, including gender, Ann Arbor stage, levels of LDH, B symptoms and international prognostic index 
Table III. Association between the expression levels of miR-10a, miR-342-3p and TIAM1 protein in tissues of patients with ENKTCL and the demographic and clinical features of the patients.

\begin{tabular}{|c|c|c|c|c|c|c|c|c|}
\hline \multirow[b]{2}{*}{$\begin{array}{l}\text { Clinical } \\
\text { feature }\end{array}$} & \multirow[b]{2}{*}{$\begin{array}{l}\text { Cases } \\
\text { (no.) }\end{array}$} & \multicolumn{2}{|c|}{ miR-10a } & \multicolumn{2}{|c|}{ miR-342-3p } & \multicolumn{3}{|c|}{ TIAM1 } \\
\hline & & $\begin{array}{l}\text { Expression } \\
\quad\left(\mathrm{x} 10^{-3}\right)^{\mathrm{a}}\end{array}$ & P-value ${ }^{b}$ & $\begin{array}{c}\text { Expression } \\
\quad\left(\mathrm{x} 10^{-3}\right)^{\mathrm{a}}\end{array}$ & P-value ${ }^{b}$ & $\begin{array}{l}\text { Cases } \\
\text { (no.) }\end{array}$ & $\begin{array}{c}\text { TIAM1+ } \\
\text { cases (no.) }\end{array}$ & P-value \\
\hline Age (years) & & & 0.02 & & 0.97 & & & 0.34 \\
\hline$<60$ & 13 & $4.97 \pm 1.13$ & & $2.08 \pm 0.27$ & & 17 & 10 & \\
\hline$\geq 60$ & 2 & $1.46 \pm 0.64$ & & $2.05 \pm 0.73$ & & 4 & 1 & \\
\hline Gender & & & 0.58 & & 0.70 & & & 0.92 \\
\hline Male & 12 & $4.03 \pm 1.02$ & & $2.02 \pm 0.28$ & & 17 & 9 & \\
\hline Female & 3 & $6.41 \pm 3.43$ & & $2.29 \pm 0.56$ & & 4 & 2 & \\
\hline Ann Arbor stage & & & 0.98 & & 0.22 & & & 0.02 \\
\hline I-II & 6 & $4.46 \pm 1.98$ & & $1.68 \pm 0.41$ & & 9 & 2 & \\
\hline III-IV & 9 & $4.54 \pm 1.20$ & & $2.34 \pm 0.28$ & & 12 & 9 & \\
\hline LDH levels & & & 0.93 & & 0.90 & & & 0.51 \\
\hline High & 6 & $4.39 \pm 1.72$ & & $2.12 \pm 0.44$ & & 10 & 6 & \\
\hline Normal & 9 & $4.59 \pm 1.36$ & & $2.05 \pm 0.3$ & & 11 & 5 & \\
\hline B symptoms & & & 0.06 & & 0.88 & & & 0.53 \\
\hline Positive & 8 & $6.28 \pm 1.53$ & & $2.11 \pm 0.32$ & & 12 & 7 & \\
\hline Negative & 7 & $2.48 \pm 0.93$ & & $2.04 \pm 0.39$ & & 9 & 4 & \\
\hline IPI (score) & & & 0.61 & & 0.21 & & & 0.02 \\
\hline $0-2$ & 7 & $3.90 \pm 1.77$ & & $1.74 \pm 0.35$ & & 9 & 2 & \\
\hline $3-5$ & 8 & $5.04 \pm 1.24$ & & $2.37 \pm 0.32$ & & 12 & 9 & \\
\hline
\end{tabular}

${ }^{\mathrm{a}}$ Expression measured relative to U6; ${ }^{\mathrm{b}}$ Student's t-test; ${ }^{\mathrm{c}} \chi^{2}$ test. miR, microRNA; TIAM1, T-lymphoma invasion and metastasis inducing factor 1; ENKTCL, extranodal natural killer/T-cell lymphoma, nasal type; LDH, lactate dehydrogenase; IPI, international prognostic index.
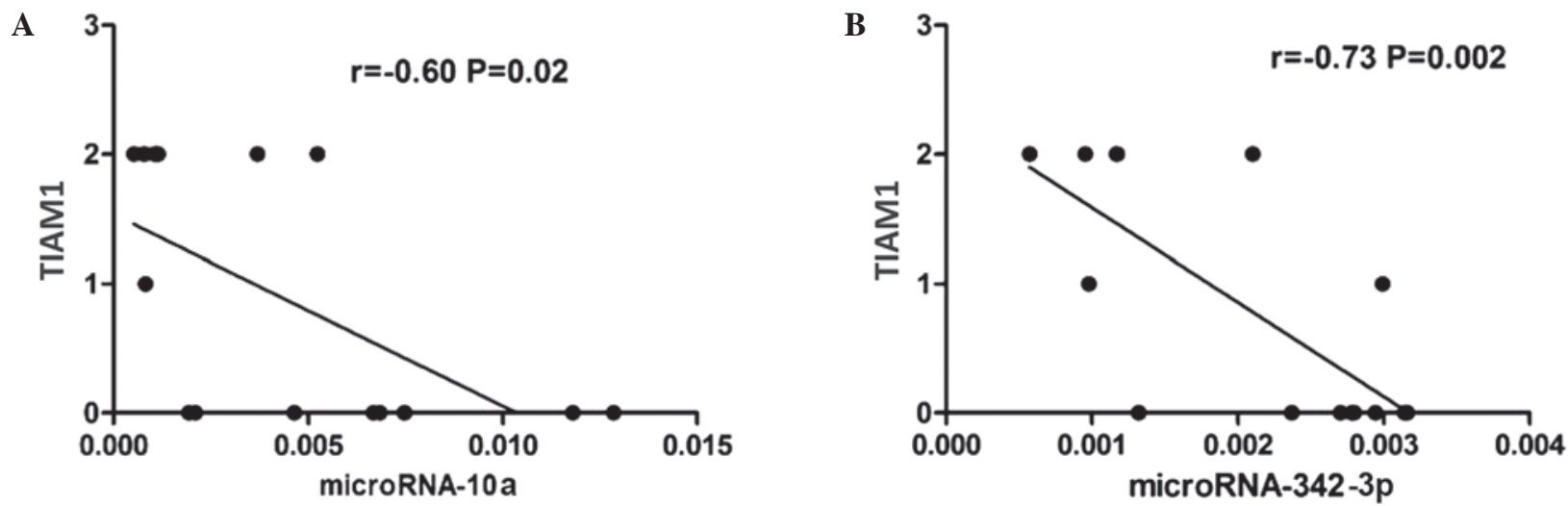

Figure 3. Correlation between the expression levels of (A) microRNA-10a and (B) microRNA-342-3p and the protein expression levels of TIAM1 in formalin-fixed paraffin-embedded tissues of patients with extranodal natural killer/T-cell lymphoma, nasal type. TIAM1, T-lymphoma invasion and metastasis inducing factor 1 .

(IPI) score (Table III). The expression levels of miR-342-3p in ENKTCL FFPE tissues was not correlated with any demographic or clinical features of the patients.

Immunohistochemistry and correlation between the expression levels of TIAM1 protein and the demographic and clinical features of patients with ENKTCL. Tiam1 protein was expressed in 11 ENKTCL samples (52.4\%) and in 1 of 10 paired samples of normal and reactive lymph node hyperplasia
(10\%), where its expression levels were low (Fig. 2). The intensity of TIAM1 protein expression detected in the ENKTCL tissues is listed in Table II. The protein expression levels of TIAM1 in ENKTCL FFPE tissues were positively correlated with Ann Arbor stage and IPI score ( $\mathrm{P}=0.02$; Table III), but no significant association was observed with any other demographic or clinical features of the patients. These results suggest that TIAM1 protein may be involved in the pathogenesis of ENKTCL. 
Correlations between the expression levels of $\mathrm{miR}-10 \mathrm{a}$, miR-342-3p and TIAM1 protein in ENKTCL FFPE tissues. Expression of TIAM1 protein was detected in the FFPE tissues of 7 of the 15 patients with ENKTCL analyzed (47\%). In these patients, the expression levels of miR-10a and miR-342-3p appeared to be inversely correlated with the protein expression levels of TIAM1 (Spearman's $\mathrm{r}=-0.60$ and -0.73 for miR-10a and miR-342-3p, respectively; $\mathrm{P}=0.02$; Fig. 3).

\section{Discussion}

Previous studies have demonstrated that the expression levels of several miRNAs are downregulated in ENKTCL FFPE tissues, which is considered to contribute to the pathogenesis of the tumor by the loss of the suppressive effects that these miRNAs normally exert on their target genes (1,21-25). In a study by $\mathrm{Ng}$ et al (1), the expression levels of miR-10a, miR-22, miR-340, miR-342-3p and miR-590-5p appeared to be downregulated in ENKTCL FFPE tissues, compared with normal NK cells, according to the results of human miRNA microarray analysis. However, these findings were not further validated by qPCR.

The qPCR data of the present study are consistent with the previous miRNA microarray results reported by $\mathrm{Ng}$ et al (1), confirming that miR-10a and miR-342-3p are downregulated in ENKTCL FFPE tissues, compared with normal NK cells. However, in the present study, the expression levels of miR-22, miR-340 and miR-590-5p did not differ significantly between ENKTCL tissues and normal NK cells. Furthermore, the expression levels of miR-10a in the ENKTCL FFPE tissues correlated with the patients' age, but the expression levels of miR-342-3p did not correlate with any demographic or clinical feature of the patients. Therefore, further studies are required to validate the potential participation of miR-10a and miR-342-3p in the pathogenesis of ENKTCL.

Previous studies have identified several target genes of miR-10a, including high-mobility group A2 (26), cell adhesion molecule L1-like (27), homeobox (HOX)A1 (28) and HOXD4 (29), which are involved in cellular differentiation, growth, migration and invasion in various pathophysiological processes (26-29). Other studies have demonstrated that the expression of miR-342-3p is downregulated in the bloodand tumor tissues of patients with cancer, including colorectal cancer (30), clinical glioblastoma multiforme (31), breast tumor (32), acute lymphoblastic leukemia (33) and Sézary syndrome (34). In addition, miR-342-3p has been previously demonstrated to be involved in cell differentiation (35), growth $(36,37)$, invasion $(37)$ and response to chemotherapy $(38,39)$ in cancer cells.

Previous studies have suggested that multiple miRNAs may suppress the expression of the same target gene by directly targeting its $3^{\prime}$ untranslated region (40). Furthermore, previous bioinformatic analysis indicated that miR-10a and miRNA-342-3p target the TIAM1 gene (1).

Tiaml has been identified as a guanine nucleotide exchange factor that exchanges guanosine diphosphate for GTP in Rho-like GTPases, thereby activating them. This process leads to the activation of a signaling pathway that stimulates the c-Jun $\mathrm{N}$-terminal kinase, p38 mitogen-activated protein kinase and extracellular signal-regulated kinase pathways, which results in the regulation of the expression of genes involved in cellular migration, invasion and metastasis $(4,5)$. To date, overexpression of TIAM1 has been reported in various types of tumor tissue, including head and neck (5), esophageal (6), colorectal (7), gallbladder (8), renal cell (9), nasopharyngeal (10), hepatocellular (11) and prostate carcinoma (12). In addition, overexpression of TIAM1 has been suggested to be involved in tumor progression via lymphangiogenesis $(13)$, apoptosis $(14,15)$, invasion and migration (16-18). However, the expression of TIAM1 in ENKTCL FFPE tissue and its association with clinical features of patients with ENKTCL remain unclear.

The immunohistochemistry data of the present study demonstrated that TIAM1 protein was overexpressed in ENKTCL FFPE tissues, compared with normal and reactive lymph node hyperplasia FFPE tissues, which is in agreement with the results of previous studies on various malignancies (4-6,8-13). The overexpression of TIAM1 in the ENKTCL cases analyzed in the present study correlated with the Ann Arbor stage and IPI score of the tumors. However, no significant association was observed between the protein expression levels of TIAM1 and any other demographic and clinical characteristics of the patients, including age, gender, levels of LDH and B symptoms. Overall, the results of the present study suggest that TIAM1 may be involved in the pathogenesis of ENKTCL. However, due to the small sample size of the present study, further studies involving a larger number of cases of ENKTCL are required in order to confirm these findings.

In conclusion, the results of the present study suggest that reduced expression of $\mathrm{miR}-10 \mathrm{a}$ and $\mathrm{miR}-342-3 \mathrm{p}$ and overexpression of TIAM1 protein may be involved in the progression of ENKTCL. Additional in vitro and in vivo studies are required to further elucidate the potential role and mechanism of action of these molecules in the development of ENKTCL.

\section{Acknowledgements}

The present study was funded by the National Natural Science Foundation of China (Beijing, China; grant no. 81201872), the Natural Science Foundation of Fujian Province (Fuzhou, China; grant no. 2013J01308), the Fujian Provincial Health Bureau Youth Research Projects (Fuzhou, China; grant no. 2011-1-12), the Medical Elite Cultivation Program of Fujian Province (Fuzhou, China; grant no. 2015-ZQN-JC-18) and the Middle Age and Young Teacher Education and Research Program of Fujian Province (Fuzhou, China ;grant no. JA14133).

\section{References}

1. Ng SB, Yan J, Huang G, Selvarajan V, Tay JL, Lin B, Bi C, Tan J, Kwong YL, Shimizu N, et al: Dysregulated microRNAs affect pathways and targets of biologic relevance in nasal-type natural killer/T-cell lymphoma. Blood 118: 4919-4929, 2011.

2. Kim SJ, Jung HA, Chuang SS, Hong H, Guo CC, Cao J, Hong XN, Suzuki R, Kang HJ, Won JH, et al; Asia Lymphoma Study Group: Extranodal natural killer/T-cell lymphoma involving the gastrointestinal tract: Analysis of clinical features and outcomes from the Asia Lymphoma Study Group. J Hematol Oncol 6: 86, 2013.

3. Esquela-Kerscher A and Slack FJ: Oncomirs-microRNAs with a role in cancer. Nat Rev Cancer 6: 259-269, 2006.

4. Cook DR, Rossman KL and Der CJ: Rho guanine nucleotide exchange factors: Regulators of Rho GTPase activity in development and disease. Oncogene 33: 4021-4035, 2014.

5. Wang S, Li S, Yang X, Yang S, Liu S, Liu B and Liu J: Elevated expression of T-lymphoma invasion and metastasis inducing factor 1 in squamous-cell carcinoma of the head and neck and its clinical significance. Eur J Cancer 50: 379-387, 2014. 
6. Liu H, Shi G, Liu X, Wu H, Fan Q and Wang X: Overexpression of Tiam1 predicts poor prognosis in patients with esophageal squamous cell carcinoma. Oncol Rep 25: 841-848, 2011.

7. Jin H, Li T, Ding Y, Deng Y, Zhang W, Yang H, Zhou J, Liu C, Lin J and Ding Y: Methylation status of T-lymphoma invasion and metastasis 1 promoter and its overexpression in colorectal cancer. Hum Pathol 42: 541-551, 2011.

8. Du X, Wang S, Lu J, Wang Q, Song N, Yang T, Dong R, Zang L, Yang Y, Wu T and Wang C: Clinical value of Tiam1-Rac1 signaling in primary gallbladder carcinoma. Med Oncol 29:1873-1878, 2012

9. Zhao L, Liu Y, Sun X, He M and Ding Y: Overexpression of $\mathrm{T}$ lymphoma invasion and metastasis 1 predict renal cell carcinoma metastasis and overall patient survival. J Cancer Res Clin Oncol 137: 393-398, 2011

10. Qi Y, Huang B, Yu L, Wang Q, Lan G and Zhang Q: Prognostic value of Tiam 1 and Racl overexpression in nasopharyngeal carcinoma. ORL J Otorhinolaryngol Relat Spec 71: 163-171, 2009

11. Ding Y, Chen B, Wang S, Zhao L, Chen J, Ding Y, Chen L and Luo R: Overexpression of Tiam1 in hepatocellular carcinomas predicts poor prognosis of HCC patients. Int J Cancer 124: 653-658, 2009

12. Engers R, Mueller M, Walter A, Collard JG, Willers R and Gabbert HE: Prognostic relevance of Tiam1 protein expression in prostate carcinomas. Br J Cancer 95: 1081-1086, 2006.

13. Zhong D, Li Y, Peng Q, Zhou J, Zhou Q, Zhang R and Liang H: Expression of Tiam1 and VEGF-C correlates with lymphangiogenesis in human colorectal carcinoma. Cancer Biol Ther 8 : 689-695, 2009

14. Cao-Hong, Shibayama-Imazu T, Masuda Y, Shinki T, Nakajo S and Nakaya K: Involvement of Tiam1 in apoptosis induced by bufalin in HeLa cells. Anticancer Res 27: 245-249, 2007.

15. Minard ME, Ellis LM and Gallick GE: Tiam1 regulates cell adhesion, migration and apoptosis in colon tumor cells. Clin Exp Metastasis 23: 301-313, 2006

16. Adam L, Vadlamudi RK, McCrea P and Kumar R: Tiam1 overexpression potentiates heregulin-induced lymphoid enhancer factor- $1 / \beta$-catenin nuclear signaling in breast cancer cells by modulating the intercellular stability. J Biol Chem 276: 28443-28450, 2001

17. Engers R, Springer E, Michiels F, Collard JG and Gabbert HE Rac affects invasion of human renal cell carcinomas by upregulating tissue inhibitor of metalloproteinases (TIMP)-1 and TIMP-2 expression. J Biol Chem 276: 41889-41897, 2001.

18. Bourguignon LY, Zhu H, Shao L and Chen YW: Ankyrin-Tiam1 interaction promotes Racl signaling and metastatic breast tumor cell invasion and migration. J Cell Biol 150: $177-191,2000$

19. Swerdlow SH, Campo E, Harris NL, Jaffe ES, Pileri SA, Stein H, Thiele J and Vardiman JW (eds): WHO Classification of Tumours of Haematopoietic and Lymphoid Tissue. 4th edition. IARCPress, Lyon, France, pp179-350, 2008.

20. Bierman PJ, Harris N and Armitage JO: Non-Hodgkin's lymphoma. In: Cecil Medicine. Goldman L and Ausiello D (eds) 23rd edition. Saunders Elsevier, Philadelphia, pp1408-1425, 2008.

21. Komabayashi Y, Kishibe K, Nagato T, Ueda S, Takahara M and Harabuchi Y: Downregulation of miR-15a due to LMP1 promotes cell proliferation and predicts poor prognosis in nasal NK/T-cell lymphoma. Am J Hematol 89: 25-33, 2014.

22. Huang HB, Zhan R, Wu SQ, Xu ZZ and Fan LP: Expression of MCL-1 and microRNA-29a in extranodal NK/T-cell lymphoma tissue. Zhongguo Shi Yan Xue Ye Xue Za Zhi 21: 95-98, 2013 (In Chinese)

23. Motsch N, Alles J, Imig J, Zhu J, Barth S, Reineke T, Tinguely M, Cogliatti S, Dueck A, Meister G, et al: MicroRNA profiling of Epstein-Barr virus-associated NK/T-cell lymphomas by deep sequencing. PLoS One 7: e42193, 2012.

24. Ti HJ, Nong L, Wang W, Zhang S and Li T: Expression of microRNA in extranodal NK/T cell lymphoma, nasal type. Zhonghua Bing Li Xue Za Zhi 40: 610-615, 2011 (In Chinese).
25. Paik JH, Jang JY, Jeon YK, Kim WY, Kim TM, Heo DS and Kim CW: MicroRNA-146a downregulates NFאB activity via targeting TRAF6 and functions as a tumor suppressor having strong prognostic implications in NK/T cell lymphoma. Clin Cancer Res 17: 4761-4771, 2011.

26. Zhu S, Deng S, Ma Q, Zhang T, Jia C, Zhuo D, Yang F, Wei J, Wang L, Dykxhoorn DM, et al: MicroRNA-10A* and MicroRNA-21 modulate endothelial progenitor cell senescence via suppressing high-mobility group A2. Circ Res 112 : 152-164, 2013.

27. Long MJ, Wu FX, Li P, Liu M, Li X and Tang H: MicroRNA-10a targets CHL1 and promotes cell growth, migration and invasion in human cervical cancer cells. Cancer Lett 324: 186-196, 2012.

28. Ohuchida K, Mizumoto K, Lin C, Yamaguchi H, Ohtsuka T, Sato N, Toma H, Nakamura M, Nagai E, Hashizume M and Tanaka M: MicroRNA-10a is overexpressed in human pancreatic cancer and involved in its invasiveness partially via suppression of the HOXA1 gene. Ann Surg Oncol 19: 2394-2402, 2012

29. Tan Y, Zhang B, Wu T, Skogerbø G, Zhu X, Guo X, He S and Chen R: Transcriptional inhibiton of Hoxd4 expression by miRNA-10a in human breast cancer cells. BMC Mol Biol 10: $12,2009$.

30. Grady WM, Parkin RK, Mitchell PS, Lee JH, Kim YH, Tsuchiya KD, Washington MK, Paraskeva C, Willson JK, $\mathrm{Kaz} \mathrm{AM}$, et al: Epigenetic silencing of the intronic microRNA hsa-miR-342 and its host gene EVL in colorectal cancer. Oncogene 27: 3880-3888, 2008.

31. Haapa-Paananen S, Chen P, Hellström K, Kohonen P, Hautaniemi S, Kallioniemi O and Perälä M: Functional profiling of precursor MicroRNAs identifies MicroRNAs essential for glioma proliferation. PLoS One 8: e60930, 2013.

32. Buffa FM, Camps C, Winchester L, Snell CE, Gee HE, Sheldon H, Taylor M, Harris AL and Ragoussis J: MicroRNA-associated progression pathways and potential therapeutic targets identified by integrated mRNA and microRNA expression profiling in breast cancer. Cancer Res 71: 5635-5645, 2011

33. Xu L, Liang YN, Luo XQ, Liu XD and Guo HX: Association of miRNAs expression profiles with prognosis and relapse in childhood acute lymphoblastic leukemia. Zhonghua Xue Ye Xue Za Zhi 32: 178-181, 2011 (In Chinese).

34. Ballabio E, Mitchell T, van Kester MS, Taylor S, Dunlop HM, Chi J, Tosi I, Vermeer MH, Tramonti D, Saunders NJ, et al: MicroRNA expression in Sezary syndrome: Identification, function, and diagnostic potential. Blood 116: 1105-1113, 2010.

35. Wu Y, Li XF, Yang JH, Liao XY and Chen YZ: microRNAs expression profile in acute promyelocytic leukemia cell differentiation induced by all-trans retinoic acid and arsenic trioxide. Zhonghua Xue Ye Xue Za Zhi 33: 546-551, 2012 (In Chinese).

36. Leivonen SK, Sahlberg KK, Mäkelä R, Due EU, Kallioniemi O, Børresen-Dale AL and Perälä M: High-throughput screens identify microRNAs essential for HER2 positive breast cancer cell growth. Mol Oncol 8: 93-104, 2014.

37. Wang H, Wu J, Meng X, Ying X, Zuo Y, Liu R, Pan Z, Kang T and Huang W: MicroRNA-342 inhibits colorectal cancer cell proliferation and invasion by directly targeting DNA methyltransferase 1. Carcinogenesis 32: 1033-1042, 2011.

38. He YJ, Wu JZ, Ji MH, Ma T, Qiao EQ, Ma R and Tang JH: miR-342 is associated with estrogen receptor- $\alpha$ expression and response to tamoxifen in breast cancer. Exp Ther Med 5: 813-818, 2013

39. Kim CH, Kim HK, Rettig RL, Kim J, Lee ET, Aprelikova O, Choi IJ, Munroe DJ and Green JE: miRNA signature associated with outcome of gastric cancer patients following chemotherapy. BMC Med Genomics 4: 79, 2011

40. Wu S, Huang S, Ding J, Zhao Y, Liang L, Liu T, Zhan R and He X: Multiple microRNAs modulate p21Cip1/Waf1 expression by directly targeting its $3^{\prime}$ untranslated region. Oncogene 29 : 2302-2308, 2010 